\title{
Effects of acupuncture in diabetes neuropathy: case
} report

\begin{abstract}
Type II (TII) diabetes therapy is an economic and scientific global health challenge. Despite advances made in conventional diabetic treatment for varied stages of disease, these treatments still cannot prevent diabetic complications and can cause severe side effects without curative guarantee for the long term. To this date, acupuncture has proven beneficial for palliative purposes and for treatment of diabetic neuropathy. We present a case of a $69 \mathrm{y} / \mathrm{o}$ male patient who suffered foot injury/infection/neuropathy and chose to forego standard diabetic medical care in favor of therapeutic acupuncture. The favorable outcomes from this case (blood glucose from $220 \mathrm{mg} / \mathrm{dL}$ reduced to 125 $\mathrm{mg} / \mathrm{dL}$, HA1c 9.1 reduced to 6.7 total during three years period) give way to the idea of potentially establishing standard acupunctural protocols via clinical trials for the treatment of TII diabetes.
\end{abstract}

Keywords: type 2 diabetes, acupuncture, alternative medicine, palliative care
Volume I Issue I - 2017

\author{
Lu Guang Luo, $1,2,3$ Shuo Wang, ${ }^{3}$ Eric Xiao, ${ }^{3}$ \\ John Z Luo ${ }^{4}$ \\ 'Boston University School of Medicine, USA \\ ${ }^{2}$ Roger Williams Medical Center, USA \\ ${ }^{3}$ The Center for Natural Healing, USA \\ ${ }^{4}$ Insure Health, Inc, USA
}

Correspondence: Lu Guang Luo, The Center of Stem Cell Biology, Department of Research, Roger Williams Hospital, Boston University School of Medicine, Providence, USA, RI 02908,

Email mycenterfornaturalhealing@gmail.com, Igluo@BU.edu

Received: June 24, 2017 | Published: July 26, 2017

\section{Introduction}

Each year, billions of dollars are spent on conventional pharmacologic treatments for TII diabetes in the United States, but such treatments are not always effective and often lead to a range of adverse reactions (e.g., hypoglycemia). ${ }^{1}$ Metformin is still the preferred first-line agent for most TI diabetes patients; though most patients require multi-agent therapies to maintain stable blood glucose levels in the long term. Traditional Chinese Medicine (TCM) has recently become attractive to western healthcare systems because of its low costs as well as its comprehensive, holistic approach to preventing and managing diabetes symptoms. These treatments are often a combination of herbal, acupunctural, dietary, and exercisebased therapies.

For acupunctural therapies, there are several prominent theories that attempt to explain the physiological workings of this treatment. One such theory proposes that the human body mimics a biological computer where organs are the headquarters and the body surface is the keyboard. ${ }^{2}$ Both are wired using acupuncture meridians, through which physiological instructions can be 'sent'. Another theory, named the Connective Tissue Stretch Theory, hypothesizes that acupuncture meridians may be associated with connective tissues, as they appear to be preferentially located along connective-tissue planes between muscles and/or bones. ${ }^{3}$

Past scholars have noted that more than 80 percent of acupuncture points on the arm are located along connective-tissue planes. More biochemically-focused explanations of acupunctural mechanisms posit that certain insertion points can cause an increase in ATP production. In what is termed the ATP/ADP transition model, the released ATP will act as a transmitter that will be rapidly degraded to adenosine by several ectonucleotidases before re-uptake into cells. ${ }^{4}$ The adenosine then acts as a pain suppressor via binding to Gi-coupled A1-adenosine receptors.

But while the biochemical mechanism of acupuncture therapy remains unclear, its clinical benefits have been documented in a number of diseases. Clinical studies dating back to the mid-late 20th century across China, Japan and South Korea have shown that acupuncture therapy can help reduce fasting blood glucose, insulin resistance, body mass index (BMI), and cholesterol levels. ${ }^{5-8}$ The efficacy of acupuncture therapy in treating secondary symptoms of diabetes, including peripheral diabetic neuropathy, has also been reported in human studies. ${ }^{9}{ }^{10}$ More than two dozen clinical trials have concluded that acupuncture therapy, in conjunction with standard hypoglycemic drugs, is effective in improving diabetic neuropathy symptoms compared to treatment regimens consisting solely of such pharmacological agents. ${ }^{9}$

In our clinical practice, many diabetic patients (including Type I [TI and TII) have experienced significant improvement in their symptoms, particularly in the form of requiring lower insulin dosages (TI) or no longer requiring insulin injections altogether (TII). The Zhongwan and Zusanli (ST36) insertion points, located at the stomach and front leg respectively, are standard targets in TCM diabetes treatment and help to regulate $q i$ flow and improve spleen and stomach function. Other common insertion points include, Taichong or LR3 (foot), Yinlingquan or SP9 (inner leg), Sanyinjiao or SP6 (inner leg), Xuanzhong or GB39 (outer leg), Quchi or LI11 (forearm), Zulinqi or GB41 (gall bladder), and Nei Guang or PC 5 (wrist). ${ }^{11,12}$

Still, a general lack of knowledge of TCM in western medicine, which has led to methodological limitations and inconclusive or contradictory results in clinical studies, has prevented its integration into conventional diabetic treatment paradigms as an alternative medicine for diabetic treatment. A similar lack of research exists in the realm of TCM in palliative care for diabetic patients. We present a case of a $69 \mathrm{y} / 0$ male diagnosed with TII diabetes who broke his right leg in a car accident and suffered an infection and various forms of inflammation and neuropathy as a result. This patient declined conventional diabetic treatment in favor of acupuncture treatment, and we report encouraging results from this case.

\section{Case description}

A $69 \mathrm{y} / \mathrm{o}$ male with significant left ankle fracture injured from car accident. His past surgical history was three ankle fracture surgeries 
with two screws and nails to fixation of fracture of Tibia and Fibula. His surgery process was successful but his diabetic condition caused his ankle inflammation and neuropathy in severe condition. After four months of antibiotic (Cefazolin AND tobramycin plus penicillin) treatment, his ankle swollen, pain and hot feeling still sever and come to ask acupuncture treatment. After six months treatment, his ankle's inflammation completely disappeared and foot function recovered (Figure $1 \& 2$ ).

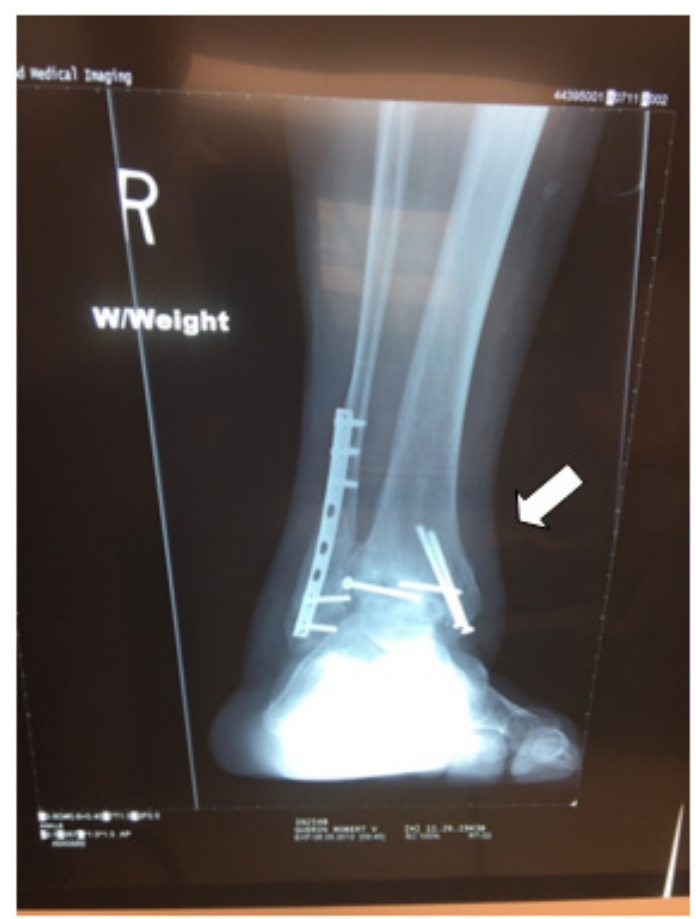

Figure I Before acupuncture treatment.

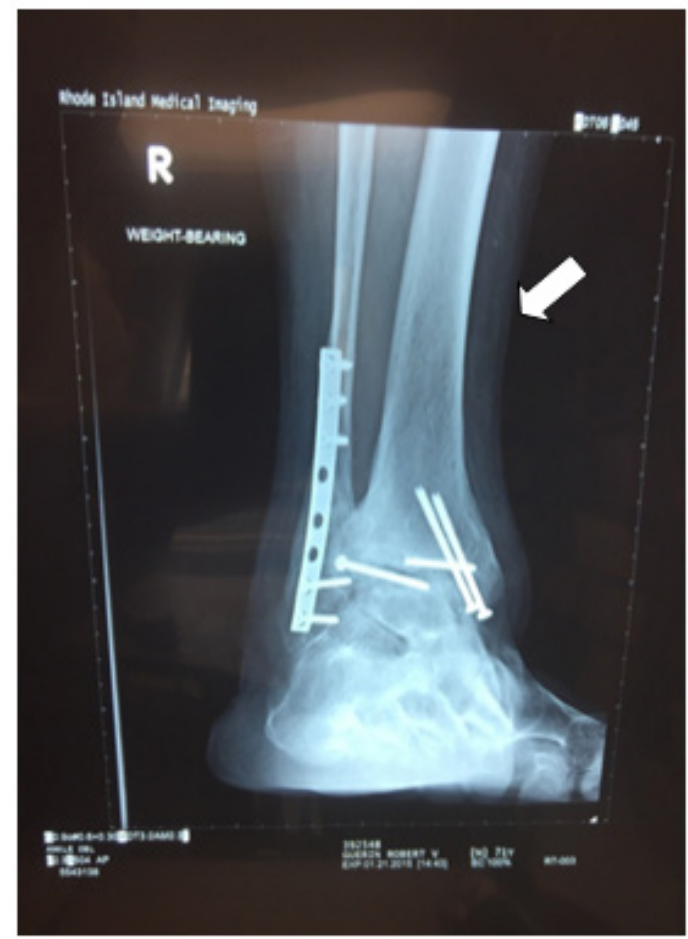

Figure 2 After acupuncture treatment.
Vascular: DP is $1 / 4$ bilateral, PT is 2/4bilateral. Capillary fill is brisk to both feet at the level of the digit. Neurological L Epicritic sensation is intact and symmetric bilateral. There is no loss of protective sensation to either foot utilizing a Symmers-Weinstein monofilament. Muscle strength appears to be intact and symmetric bilateral. His diabetic condition is consistent with diabetes blood test (fasting glucose $140 \mathrm{mg} / \mathrm{dL}$, Hemoglobin A1C 7.0). His family history was positive for high blood pressure, heart disease and diabetes. He was under diabetic medication Metformin Hydrochloride $1000 \mathrm{mg}$ and he complained about discomfort from the medication and intended to discontinue this medication. After he stopped diabetic medication, his fasting HA1c level significantly increased to 8.4, Cholesterol Total $216 \mathrm{mg} / \mathrm{dl}$, LDL $145 \mathrm{mg}$ and Glucose $173 \mathrm{mg} / \mathrm{dl}$. At this stage, patient required acupuncture treatment. After six months, HA1c level reduced to 8.1, Cholesterol Total $225 \mathrm{mg} / \mathrm{dl}$, LDL $165 \mathrm{mg}$, Glucose slight reduced to $157 \mathrm{mg} / \mathrm{dl}$. Continuing six months acupuncture treatment, his HA1c level reduced to 6.7, Cholesterol Total $201 \mathrm{mg} /$ $\mathrm{dl}$, LDL $135 \mathrm{mg}$, Glucose reduced to $133 \mathrm{mg} / \mathrm{dl}$.

Patient then stopped acupuncture treatment for one year and returned to our practice with a HA1c level of 9.1, Cholesterol Total $233 \mathrm{mg} / \mathrm{dl}$, LDL $162 \mathrm{mg}$, Glucose to $176 \mathrm{mg} / \mathrm{dl}$. After acupuncture treatment for six months, his HA1c level reduced to 8.1, Cholesterol Total $225 \mathrm{mg} / \mathrm{dl}$, LDL $165 \mathrm{mg}$, Glucose slight reduced to $157 \mathrm{mg} / \mathrm{dl}$. After one year, his HA1c level reduced to 7.6, Cholesterol Total 216 $\mathrm{mg} / \mathrm{dl}$, LDL $148 \mathrm{mg}$, Glucose slight reduced to $145 \mathrm{mg} / \mathrm{dl}$. Other lab test results are all normal. Summary as Table 1 .

Ophthalmology special report VA OD: sc20/25 NccJit-2 OS: sc20/40 NccJ2. Anterior L/C/S Right eye: Meibomian Gland Dysfunction 1+ Ptosis Conjunctiva and Sciera without injection. Left eye is same. Right eye: Cornea Tace Band keratophy (Nasal); left eye same. Right eye: Anterior Chamber Normal Depth, Quiet; left eye is same. Right eye: Iris Flat left eye is same. Right eye: Lens 1+NS, left eye is same. Posterior right eye: Nerve No disc Edema. No Disc Pallor. CDR 0.3, left eye is same. Right eye Vitreous Clear, left eye is same. Right eye Retinal Vesseis Normal Callber, left eye is same. Right eye Macula No Edema. Good foveal Reflex, left eye is same. Right eye Periphery No Holes or Tears, Attached 360 degrees, left eye is same. Imp: Mild nonproliferative diabetic retinopathy OD. Minimal NCO proliferative diabetic retinopathy is present on examination. The result has been not changed since patient started with acupuncture treatment (5 years).

Since starting on acupuncture treatment, patient never developed any symptoms of neuropathy. His injured ankle completely recovered without any motion restrictions. Patient's diabetic symptoms included polydipsia, polyphagia, and polyuria at beginning. In TCM terms, patient was diagnosed as being of a typical 'heat' type with dampness in middle Jiao and a meridian disorder in the kidney, bladder, spleen, and stomach. Acupuncture therapy consisted of targeting the following points: GB39 (outer leg), GB41 (foot), LI11 (forearm), LR3 (foot), PC5 (forearm), SP6 (inner leg), SP9 (inner leg), and ST36 (front leg). The acupuncture treatment aimed to enhance kidney, bladder and spleen function and reduce over-active stomach, as well as to reduce heat and dampness the in middle Jiao to control diabetic symptoms. In the six months before and after acupuncture treatment, patient's blood glucose level was monitored weekly and was shown to have decreased from 145 and $220 \mathrm{mg} / \mathrm{dL}$ before treatment to 127 and $165 \mathrm{mg} / \mathrm{dL}$ after treatment. The peak blood glucose level appears to have seen a more significant reduction $(55 \mathrm{mg} / \mathrm{dL})$ from before acupuncture treatment $(220 \mathrm{mg} / \mathrm{dL})$, to after treatment $(165 \mathrm{mg} /$ 
dL). In the lower blood glucose measurements, glucose levels still decreased from before $(145 \mathrm{mg} / \mathrm{dL})$ to after treatment $(127 \mathrm{mg} / \mathrm{DL})$, but this reduction is less significant $(18 \mathrm{mg} / \mathrm{dL})$. For both before and after treatment periods, the blood glucose changes ranged from 10 to $65 \mathrm{mg} / \mathrm{dL}$. Qualitatively, patient self-reported improved sleep quality, less over-eating, and less overall fatigue.

Table I Blood test results summery

\begin{tabular}{|c|c|c|c|c|c|c|c|}
\hline Indexldata & Starting & 6 Month & 6 Month & Stop one year & Starting & 6 Month & 6 Month \\
\hline $\mathrm{Halc}$ & 8.4 & 8.1 & 6.7 & & 9.1 & 8.1 & 7.6 \\
\hline Total cholesterol & $216 \mathrm{mg} / \mathrm{Dl}$ & 225 mg/DI & 201 mg/DI & & $233 \mathrm{mg} / \mathrm{DI}$ & $225 \mathrm{mg} / \mathrm{DI}$ & $216 \mathrm{mg} / \mathrm{Dl}$ \\
\hline LDL & 145 mg & $165 \mathrm{mg}$ & $135 \mathrm{mg}$ & & $162 \mathrm{mg}$ & $165 \mathrm{mg}$ & 148 mg \\
\hline Glucose & 173 mg/DI & I 57 mg/DI & | 33 mg/DI & & 176 mg/DI & I57mg/DI & | 45 mg/DI \\
\hline
\end{tabular}

\section{Discussion/conclusion}

Our case report suggests that TCM acupunctural therapy has the potential to treat a range of TII diabetes symptoms, including hyperglycemia, diabetic neuropathy, and other pain-related complications. Diet and life style change also play a critical role in acupuncture treatment. Based on the patient's clinical profile, results suggest that palliative care patients may benefit from TCM therapies as well. This treatment modality could also be sufficient to control diabetes progression and prevent symptoms of diabetic complications in ophthalmological, cardiac and renal forms in long term treatment (three years at least). However, this is only one case report and more standardized studies will be necessary for these practices to become a standard treatment option for patients.

As conventional pharmacologic treatments for endocrine disorders remains unsurpassed when it comes to proven safety and effectiveness. The clinical and economic advantages of TCM acupuncture therapy for TII still needs more than warrant further investigation into potential integration of such treatments into the western standard of TII diabetes care.

\section{Acknowledgments}

None.

\section{Conflict of interest}

Author declares no conflict of interest.

\section{References}

1. Petersen M. American Diabetes Association 2013. Economic Costs of Diabetes in the U.S. in 2012. Diabetes Care. 2013;36(4):1033-1046.

2. Dong J. The Relationship between Traditional Chinese Medicine and Modern Medicine. Evidence-Based Complementary Alternative Medicine. 2013;2013:10.
3. Mc Gechie D. The Connective Tissue Hypothesis for Acupuncture Mechanisms. Journal of Chinese Medicine. 2010;93:14-21.

4. Burnstock G. Acupuncture: A Novel Hypothesis for the Involvement of Purinergic Signalling. Med Hypotheses. 2009;73(4):470-472.

5. Qian ZR, XL Zhong, YA Fang. Traditional Chinese medicine combined with Western medicine in the treatment of diabetic peripheral neuropathy. Zhong Xi Yi Jie He Za Zhi. 1987;7(3):140-142.

6. Chen D, Gong D, Zhai Y. Clinical and Experimental Studies in Treating Diabetes Mellitus by Acupuncture. J Tradit Chin Med. 1994;14(3):163166

7. Dharmananda S. Treatment of Diabetes with Chinese Herbs and Acupuncture. Internet Journal of the Institute for Traditional Medicine and Preventative Health Care. 2003.

8. Darbandi M, Darbandi S, Owji AA, et al. Auricular or Body Acupuncture: Which One is More Effective in Reducing Abdominal Fat Mass in Iranian Men with Obesity: A Randomized Clinical Trial. J Diabetes Metab Disord. 2014;13(1):92.

9. Yamada A, Nakamura H, Kurono Y, et al. Acupuncture Treatment in a Patient with Diabetes Mellitus. Japan Society of Acupuncture and Moxibustion. 2002;52(1):49-55.

10. Chen W, Yang G, Liu B, et al. Manual Acupuncture for Treatment of Diabetic Peripheral Neuropathy: A Systematic Review of Randomized Controlled Trials. PLoS One. 2013;8(9):e73764.

11. Jeon E, Kwon H, Shin I, et al. Effect of Acupuncture on Diabetic Peripheral Neuropathy: An Uncontrolled Preliminary Study from Korea. Acupunct Med. 2014;32(4):350-352.

12. Meliani N, El Amine Dib M, Allali H, et al. Hypoglycaemic Effect of Berberis Vulgaris L. in Normal and Streptozotocin-Induced Diabetic Rats. Asian Pac J Trop Biomed. 2011;1(6):468-471. 